\title{
THE IMARA PROJECT - THE LOCAL DEPARTURE CONTROL SYSTEM FOR AIRPORTS
}

\author{
Jozef GALANDA*, Radoslav ŠULEJ, František ADAMČÍK \\ Faculty of Aeronautics, Technical University of Kosice, Rampova 7, 04121 Kosice, Slovak Republic \\ *Corresponding author. E-mail: jozef.galanda@tuke.sk
}

\begin{abstract}
The paper deals with the issue of Local Departure Control System (further only DCS) and its application in the airport environment. The authors submit their own DCS system solution supported by the Slovak Research and Development Agency under the contract no. VEGA-15-0527. The aim of the project was to design and then implement a solution that would meet the highest criteria of reliability, safety and usability of the proposed solution in the field of its deployment. The primary target groups are local carriers, airlines and airports operating in Slovakia with a special focus on regional low-cost companies and charter companies. Within the implementation of the project, algorithms of provided functions and activities of individual software modules were designed. Subsequently, the DCS hardware elements were optimally dimensioned, programmed, tested and tuned for the correct application code for the DCS system modules, as well as for CKI, Gate and PNL terminals.
\end{abstract}

Keywords: DCS; airport; PNL; CKI; Gate; Html5, Java

\section{INTRODUCTION}

The Departure Control System (further only DCS) is currently designed to automate the comprehensive management of passenger and baggage handling processes provided by the airport to individual air carriers. Passenger handling arose due to certain specificities of air travel such as limited aircraft capacity and the requirement that only passengers having a ticket purchased would board. The check-in process consists of check-in, boarding, passenger and baggage check, aircraft balancing and checking, passenger check-in (from gate to boarding) and passenger check-in after arrival. All these activities must be immediately followed up (except after the arrival). In order to ensure the smooth process of check-in the passengers' cooperation is required, as their approach to the check-in process can speed up the process, but also slow down (long queues, bump access to each phase, etc.).

The choice of airport check-in systems is mostly done by the airline. If it is a major company, taking over its own DCS system is not a big problem. Sometimes it is possible to choose an airport check-in system if this system meets the airline's requirements. The financial side also has a major impact on the choice or takeover of the system. Due to the small number of flights, it is not worth the money to buy new airport equipment for the system, but management is trying to reach a compromise with the airline. In this regard, an opportunity arose for expanding the research of the Faculty of Aeronautics in this area. At the same time, it makes it possible to establish cooperation at international level with other airlines, which will be an invaluable source of information and experience in the use of similar DCS systems and will enable the Faculty of Aeronautics to progress quickly in this field. The currently available DCS systems often do not meet the requirements of low-cost airlines and their solutions require high implementation and operational costs. Therefore, the primary goal of the implemented DCS IMARA project was to design separate (local), flexible and cost-effective DCS, specifically designed for smaller airlines. By designing and finally implementing a functional model of the L-DCS system, testing it and then implementing it for customers, the Faculty of Aeronautics can be one of the leading providers of DCS systems, thereby also making scientific and applied research visible in the Slovak Republic as well as at international level [1]. 


\section{L-DCS IMARA PROJECT}

The solution is the L-DCS system as a fully integrated multi-user and multi-host automated passenger handling system accessible from any local workstation, meeting all L-DCS security access regulations, automated check-in, full graphical visualization of available seat maps and seating process, baggage handling, boarding and automated bag tag printing, highly secure automated passenger registration system and gate access - new features for weight and balance to support aircraft balancing process and a separate flight management and editing system.

The ambition of the researchers was to transfer the results obtained within the solution of this project to the real practice of the passengers and baggage handling process of. By sharing the features of the proposed project modules, the entire passenger handling process is ensured. The output of the project is a software product with high applicability for practice with innovative key benefits that are the basis of high originality and innovativeness of the solution.

1. The primary advantage of the system is ensuring communication between modules through Internet or intranet technologies, based on the latest HTML5 and Java technology standards, and the implementation of individual modules as Internet applications in the form of active dynamic pages, ensuring system independence on hardware and system platforms.

2. The prerequisite for the deployment of the final implementation of the DCS system with supporting hardware is its physical location directly at the destination airport as a target customer, as well as supporting devices enabling communication on the local computer network, eliminating the very common problems of data availability and errors as $90 \%$ of DCS systems that operate via WAN communication lines. In local connection of communication devices, such unreliability is virtually eliminated and in the event of failure of any device, the failure is not dependent on the third party, but the failure can be eliminated as soon as possible, thereby reducing repair costs.

3. The system is designed to be fully modular and easy to configure for the specific needs of users, unlike other DCS systems that are offered as "closed", where the customer can only choose from the system features offered and their possible modification is problematic or impossible.

4. A fast and efficient check-in process and boarding with a minimum of steps to achieve the desired goal and with a view to greater passenger satisfaction and travel well-being.

5. Possibility to quickly incorporate all new changes based on IATA and AEA recommended practices.

6. The only necessary prerequisite for the implementation of the proposed LDCS system solution is the use of Open Source modern development technologies that clearly reduce project implementation costs in terms of licensing policy [2].

The primary users of the resulting solution are local carriers, airlines and airports operating in Europe with a special focus on regional low-cost and charter airlines that create self-contained subsystems at airports for cost-per-passenger savings and the ability to self-optimize the selection of the required components.

\section{L-DCS IMARA REALIZATION}

Before the implementation of the DCS system, it was necessary to algorithmize the activities of the proposed flight planning modules (PNL), passenger check-in (CKI), their boarding (GATE) and in cooperation with the partner organization the aircraft balancing module (STW). For each proposed module, we have defined all the functions that the module must provide to the operator, which had to be algorithmized to a form suitable for programming. For example, the basic functionalities of the CKI module include updating passenger check-in list data, boarding pass generation and printing, managing and updating baggage data in the passenger list, and bag tag generation and printing. Based on their analysis from the point of view of the functionality of the DCS system, these functionalities 
were then phased into a form suitable for creating the algorithms necessary for the final program implementation of the module. This part was the most important in terms of the quality and functionality of the resulting software product, because poor analysis of individual functionalities and their algorithmization will only be shown at the stage of testing the resulting software product in the form of its malfunctioning, which will result in loss of work and time devoted to it and the entire process from proposal to the program execution of the wrong part must start again. The individual proposals were additionally modified according to the backward check of the functionality of the algorithms and with the additional features. With regard to IATA recommendations for the equipment of individual DCS workstations and consultation with potential customers, we have specified the basic concept of hardware equipment for individual workplaces and the topology of connecting individual devices to a private VPN network. Within the proposed topology, we designed hardware equipment for PNL, CKI and GATE workplaces.

Our primary goal was to create a web application that does not need to be installed on the user's device and can be run from any device using a web browser because it is running on the server side. Therefore, our choice of tools for the development of the web application has narrowed down to the following selection. As a basis, we have deployed the proven LAMP platform, which is based on the core operating system CentOS Linux, the Apache HTML server supporting the modern multi-platform database system MariaDB using the modern and widely applicable PHP7 programming language and the Symfony framework, which is currently one of the largest and most comprehensive frameworks of the PHP programming language for developing large-scale applications on the server side of the application framework. For the client-side software modules of the system, we used proven JavaScript and JavaQuery languages, which are now a clear choice for front-end applications using the ReactJS framework, which has full support for linking to the Symfony backend framework. As a complementary framework to accelerate the development of graphical Interfaces applications, we use the very popular Bootstrap framework.

On this development platform, during this stage, the process of creating and debugging individual DCS system modules took place on the basis of the proposed algorithms that cover all the required DCS system functions. At the same time, within the CKI module, we have included the output of the required information for the balancing module as the number of passengers handled, the number and total weight of the luggage and the seating of passengers in the cabin sections. The resulting solution of the PNL and CKI module user interface is shown in Figure 1. Also in the process of implementing the GATE module, it was necessary to incorporate a communication interface with 2D and 3D code readers to check the passenger IDs entering the aircraft. The resulting GATE UI solution is shown in Figure 2. For the effective management and work of the database management system (DBMS) of the MariaDB database system and the individual DCS modules, we have defined the relational model of the database. In its current form, it consists of six core entities, from a flight entity containing flight information (IATA airline code, flight number, departure date, IATA airport codes, arrival time, departure time, aircraft registration plate, aircraft cabin version, occupied capacity, etc.), Pax entities containing passenger information (name, surname, destination, passenger class, seat number, restrictions, etc.), Baggage entities containing baggage information (id, owner, weight, flight, etc.), three entities containing information about each aircraft version, occupancy and seat constraints for the flight. Of course, the relational model also includes entities for managing users and logging systems for possible data repair. Using this model, we have created a physical model of the database with individual data structures ready for data input using the DBMS of the database system.

The DCS system is designed to process and evaluate mathematical calculations where high accuracy is required, with the least possible deviation in calculations, ensuring carefree communication when transmitting data with cryptographic standards at the transmission level in order to ensure data protection. For this reason, the choice of the software life cycle (Software / System Development Life Cycle) is crucial, during which analyzing requirements, design, implementation, testing, and its further development/evolution is taking place. For the implementation of the project, we used the so-called agile methodologies that respond flexibly to change, continually schedule work during development and validate outputs with users. Thanks to an agile approach, the development 
process is based on team collaboration, open team communication, customer engagement, overall flexibility and openness to change and is therefore suitable for building more complex systems.

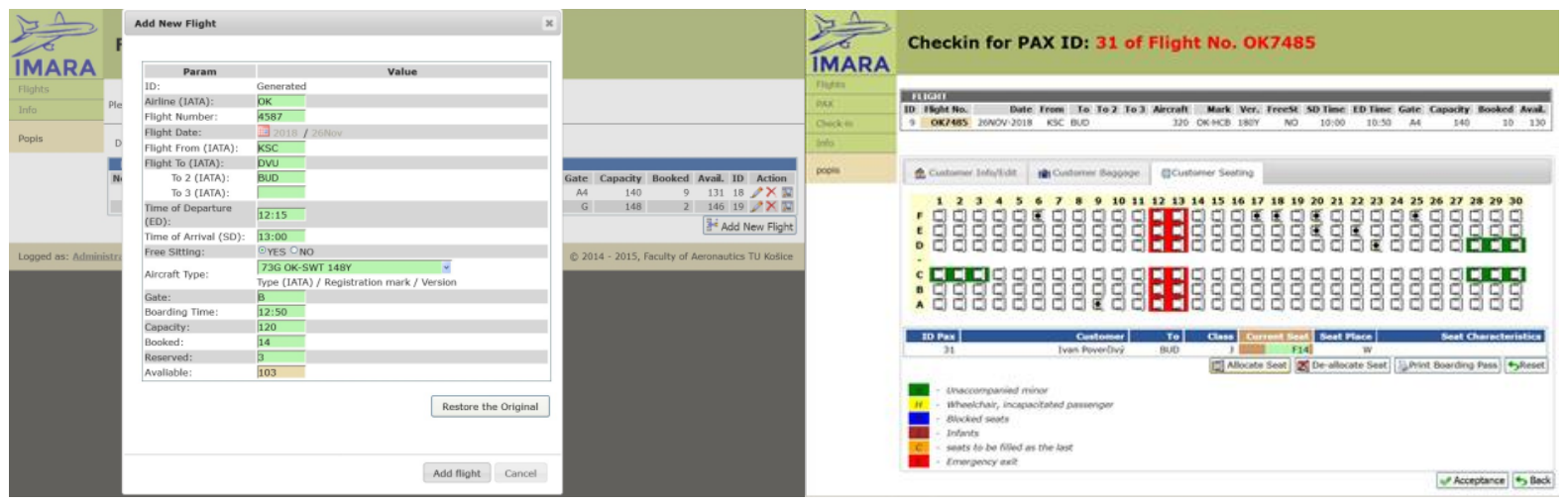

Figure 1 Communication Interface of the PNL (creating a new flight) and CKI (seat allocation) Module

The programming of the individual modules was followed by testing and tuning the DCS correctness, which is necessary to eliminate syntactic but mainly logical errors of individual software modules. In the case of non-standard behavior of the system during its debugging, it was necessary in some cases to step by step check individual modules in order to detect and correct hidden errors. Testing and debugging the modules of the software being developed is necessary to verify the functionality and accuracy of the software created.

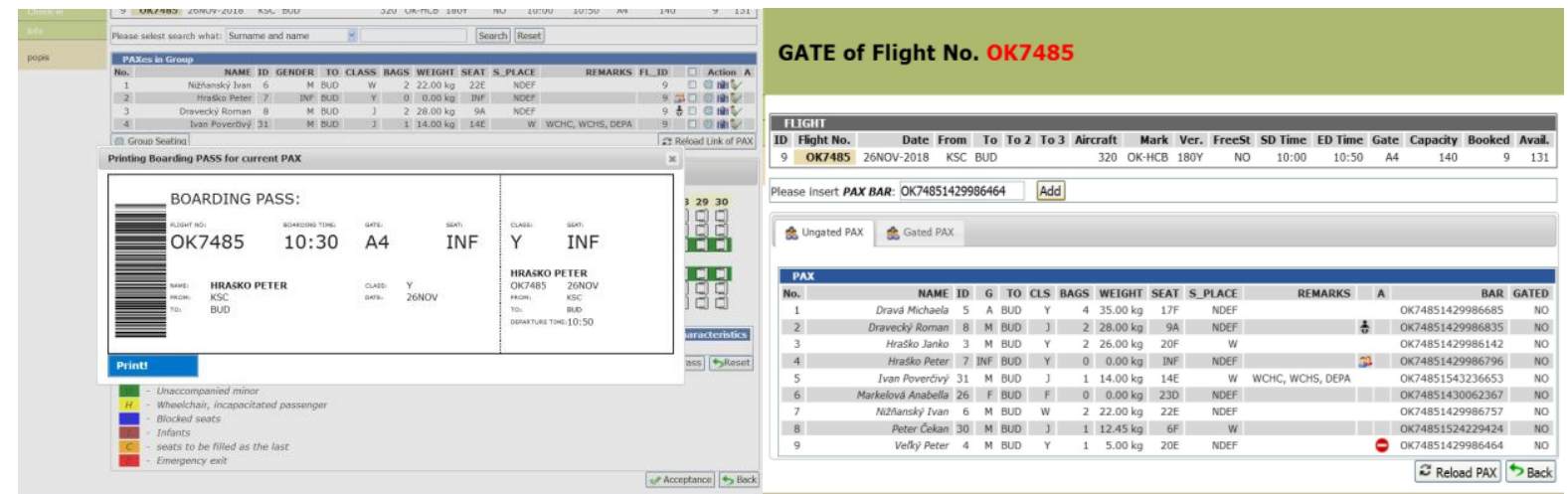

Figure 2 Communication Interface of the CKI Module (boarding pass print) and GATE Module

The implemented L-DCS simplifies the entire process of passenger handling, supporting the efficient use of agents' time. Their mistakes will be greatly reduced and passenger handling will become faster and safer. This means that the proposed more efficient processes and procedures will allow agents to better optimize their time while saving passengers' pre-flight time, which they can devote to other activities at the airport, and possibly increase airport revenue through other offered services.

\section{CONCLUSION}

The project addresses current issues in the field of handling processes at airports, examines all possible circumstances accompanying the error rate of service personnel (handling agents) during passenger and baggage handling with the aim of presenting solutions that would eliminate this error altogether or reduce it to an acceptable extent. Throughout the process, from design, through implementation, to practical testing in a particular application, effective, modern technologies, methods and practices of current practice have been used in the field of development and implementation. The project was planned as a cycle of systematic applied research to obtain information, research - the introduction of innovative elements, which led to the rapid and direct 
introduction of new knowledge into practice. We assume that, based on research findings and good practice in the project, the efficiency of the handling agents' work will be increased, the cost of training personnel and the operation of the proposed equipment system at each stage of the handling process will be reduced. We expect an increase in the employment prospects of university graduates in the labor market, acceleration of their inclusion in the airport's work process and creation of conditions for improving their quality of life. At the same time, thanks to the usability of the expected project results in the teaching process at the Faculty of Aeronautics, it will be possible to increase the readiness of the graduates of this institution for practice. Finally, by acquiring a knowledge base on handling agent responses to individual situations while working with the proposed systems to simulate real deployment with a contractor, it provides the basis for applying results in other areas of research.

\section{ACKNOWLEDGMENT}

This work was supported by the Slovak Research and Development Agency under the contract no. APVV-15-0527 - "New generation of a departure control system for airports".

\section{References}

[1] Adamčík, F. - Galanda, J. - Šulej, R. - Jenčová, E. - Jezný, M. Local modular departure control system for airports. In: New Trends in Aviation Development 2018. Danvers (USA): Institute of Electrical and Electronics Engineers. 2018. p. 8-12 [online]. - ISBN 978-1-5386-7917-3. Available at: https://doi.org/10.1109/NTAD.2018.8551639.

[2] S. Szabo, R. Sulej, J. Galanda, "Departure control system for regional and low-cost carriers", in New Trends in Civil Aviation. London: CRC Press, 2018. P. 237-244. ISBN 978-13-512-3863-2

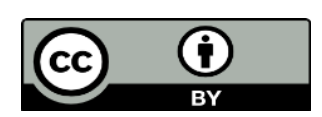

The article is licensed under a Creative Commons Attribution 4.0 International License 